\title{
Saving Energy with Buoyancy and Balance Control for Underwater Robots with Dynamic Payloads
}

\author{
Carrick Detweiler, Stefan Sosnowski, Iuliu Vasilescu, and Daniela Rus \\ Computer Science and Artificial Intelligence Lab \\ MIT, Cambridge, MA 02139, USA \\ carrick@csail.mit.edu, iuliuv@mit.edu, rus@csail.mit.edu
}

Summary. We have developed an underwater robot that can pick up and place objects during an autonomous underwater mission. This robot will have changing weight and dynamics during a mission. We have developed an efficient method for buoyancy control to achieve adaptation to weight changes without the need to increasing the work of the robot's thruster system. In this paper we describe the mechanism used for buoyancy and balance control. We also describe the adaptive control algorithms and data from physical experiments.

\section{Introduction}

We are developing underwater robot systems capable of work by manipulation such as assembling an underwater structure, collecting a sample, or pick and place of objects on the ocean floor. Our long-term goal is to develop underwater robot systems capable of sustained operation for marine studies, underwater construction, and surveillance. Consider an application where an underwater robot deploys and repositions the nodes of an underwater sensor network. As the robot picks up a module, the additional weight will contribute to changes in the robot's mass distribution and therefore its inertial characteristics in water. The robot will have to adapt to the change. One possibility is to use increased thruster power to compensate for the change. Alternatively, buoyancy control can be use to achieve adaptation to the weight change without increasing the work of the robot's thruster system.

In this paper we present a method for efficient adaptive control of underwater robots with changing body weight and dynamics. The body weight changes when the robot picks up or drops off a payload. Our approach combines balance control and buoyancy control. Specifically, we describe the architecture of two hardware modules: (1) a buoyancy control mechanism and (2) a balance control mechanism. The buoyancy control mechanism provides a power-efficient means for compensating for extra weight. It uses a piston to displace water. The balance control system provides an efficient way for controlling the robot's center of mass and overall orientation. It adjusts the robot's center of mass by moving an internal battery. Both of these systems save significant energy during the robot's mission and require energy during adjustment only. We also describe robot control algorithms that use buoyancy and balance control to response to a change in weight efficiently without increasing the thruster power and present experimental data. 
Most underwater robot systems are neutrally buoyant and do not execute tasks that require control of their buoyancy. Underwater robots that can change their buoyancy include Divebot [4] which changes its density by heating oil, SubjuGator [3 5], which uses two solenoids which regulate the amount of ballast in a buoyancy compensatory, and gliders such as the Spray gliders [6] which change their buoyancy by battery-powered hydraulic pumps in order to glide forward. Our work provides simultaneous control of balance and buoyancy to enable the pickup of a payload. Gliders are the only class of robots that also control both balance and buoyancy. However, gliders do so with different mechanisms to power their movement rather than to pick up objects stably. In our previous work [1] we demonstrated docking and coordination of two autonomous robots for long-range navigation. The robot's basic hardware and control systems are described in [8, 2, 7]. In this paper we discuss hardware and control extensions of this robot that enable adaptive buoyancy and balance control.

\section{Hardware Design for Adaptive Buoyancy and Balance}

Our autonomous underwater robot is called AMOUR V. Figure 1 shows the basic robot with the buoyancy control module extension attached on the left. The robot is neutrally buoyant. It uses five thrusters for navigation. The thrusters are arranged so that all rotational degrees of freedom can be stabilized at any time. The robot is able to swim in either horizontal or vertical orientation, with the capability of traveling forward and ascending/descending in both orientations. Travel velocity is $2.6 \mathrm{knots}$. The robot's weight

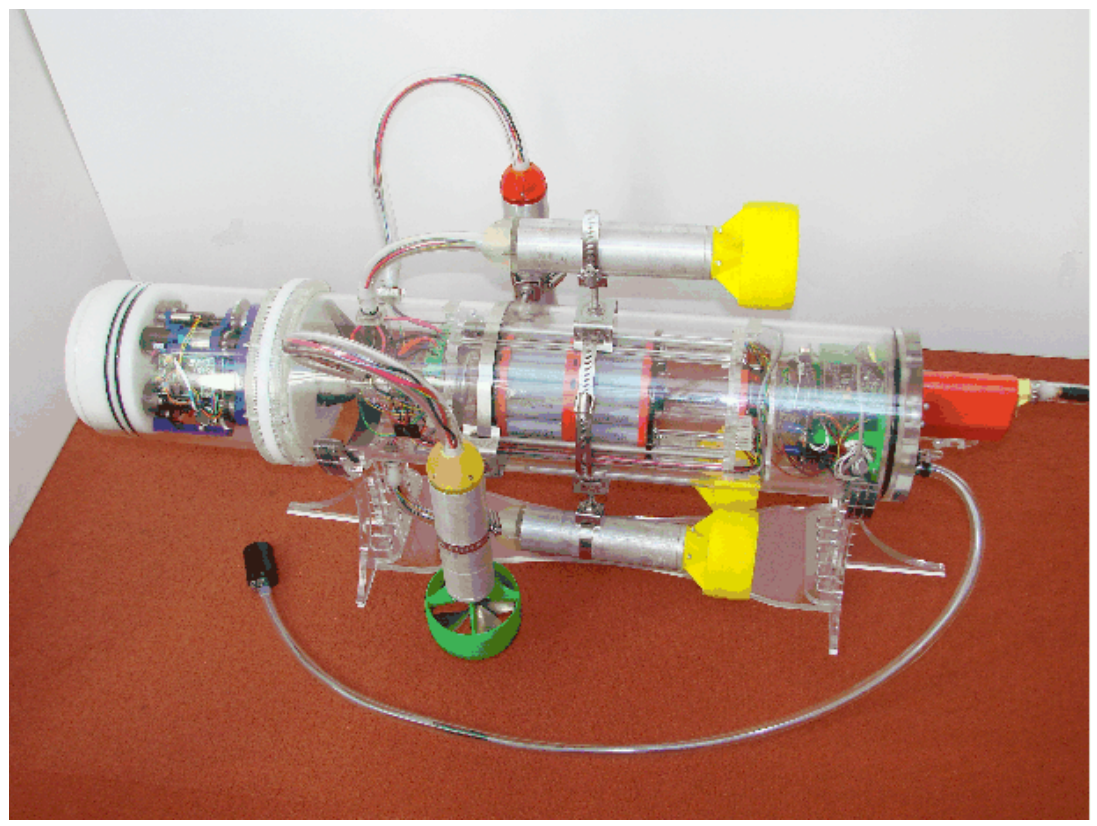

Fig. 1. AMOUR V-note the buoyancy control extension module on the left 

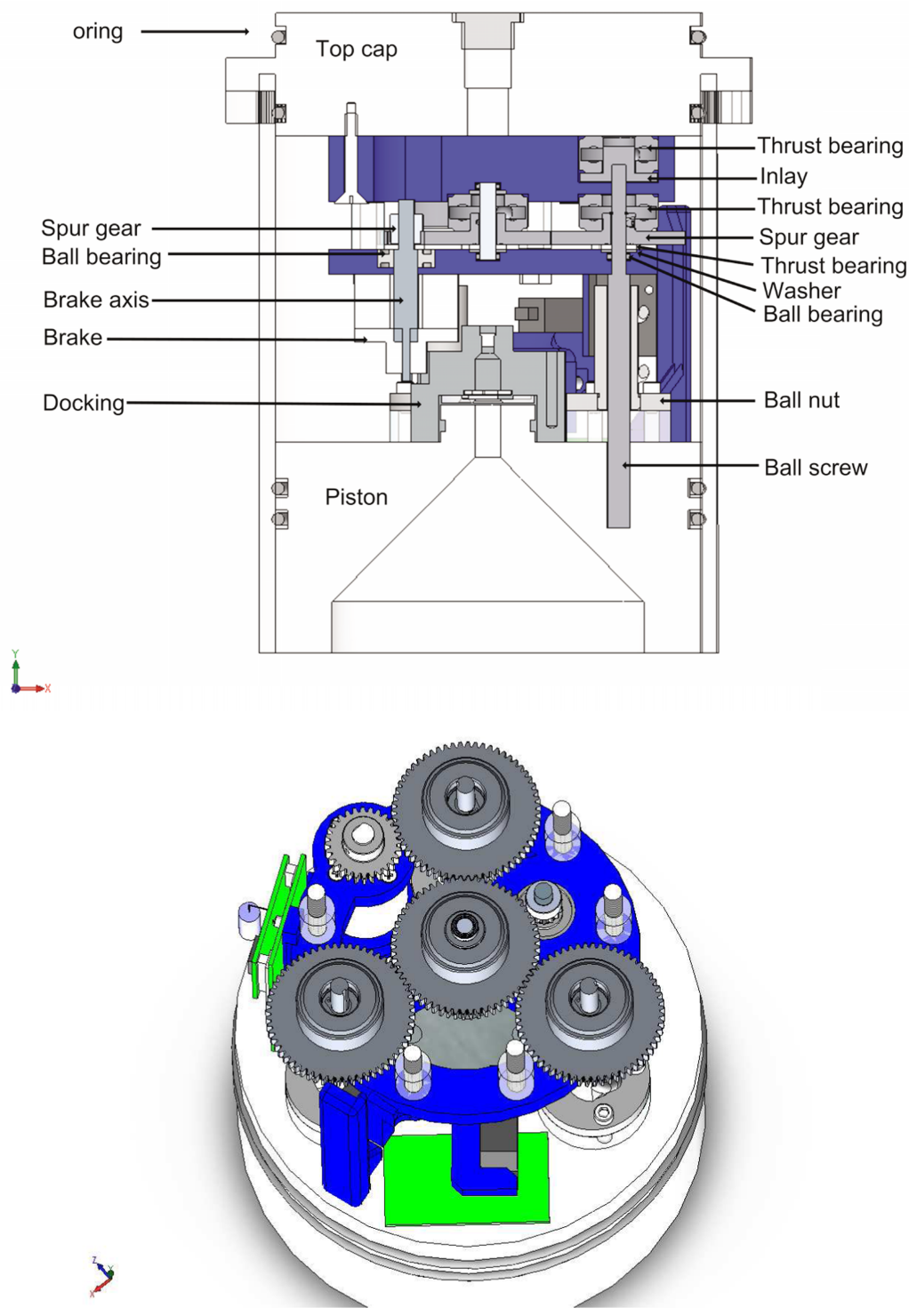

Fig. 2. (Top) Diagram of the buoyancy control mechanism. (Bottom) The gear mechanism used in the buoyancy control module.

without the buoyancy and balance modules is $19.6 \mathrm{~kg}$, its body length $0.7 \mathrm{~m}$, and its diameter of $0.18 \mathrm{~m}$. The robot's measurements with the buoyancy and balance control module is $25.5 \mathrm{~kg}$ for weight and $0.86 \mathrm{~m}$ for length. The robot includes a $3 \mathrm{D}$ IMU, a $60 \mathrm{MHz}$ ARM processor, an acoustic modem, and a $750 \mathrm{~W} \mathrm{Li-Ion} \mathrm{batter} \mathrm{package} \mathrm{which}$ gives an endurance of 8 hours. 

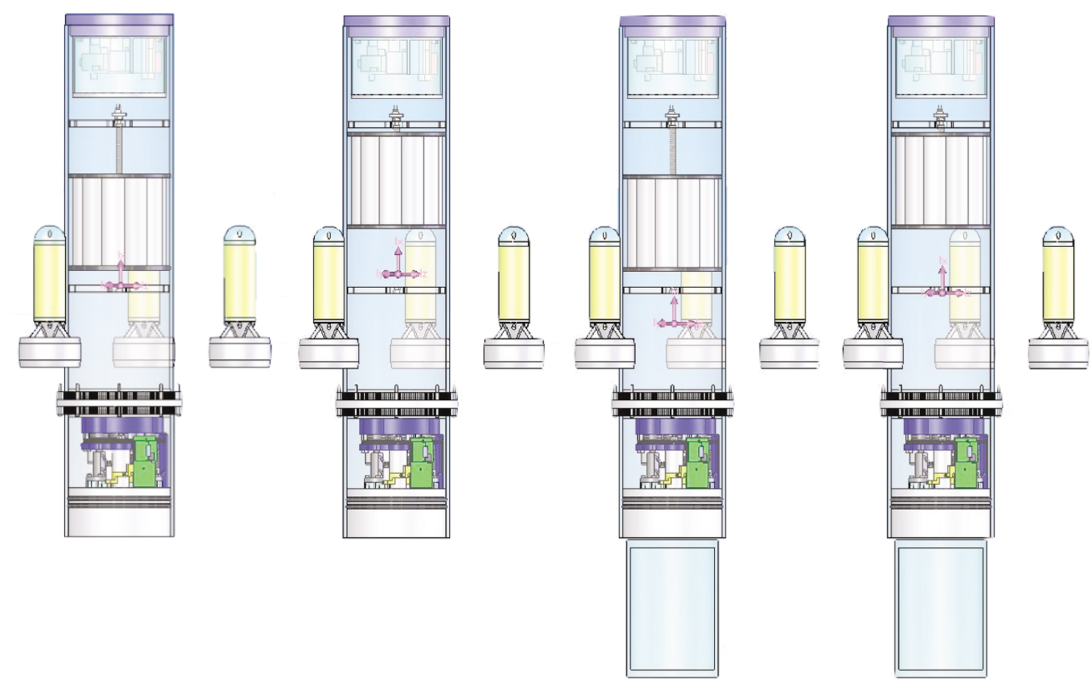

Fig. 3. Simulation of the center of mass location. Robot without weight, battery down. Robot without node, battery up. Robot with weight, battery down. Robot with weight, battery up.

We extended the robot with buoyancy and balance control mechanisms, packaged as auxiliary modules. The main design requirement for the mechanisms was to achieve adaptation to an additional payload of up to $1 \mathrm{~kg}$ within 30 seconds. The buoyancy control unit is especially useful when the robot is in vertical configuration. The balance control module is especially useful when the robot is in horizontal configuration. The robot uses its thrusters to achieve a desired depth. Extra weight would make the thrusters work harder. The role of the buoyancy control module is to bring the robot back to a neutrally buoyant state (and thus save energy). The role of the balance control module is to change the center of mass of the robot when additional weight is added or in response to the change in the buoyancy control module.

The buoyancy mechanism (see Figure 2) controls the buoyancy of the robot by moving a piston inside a cylinder. The cylinder has a $16.5 \mathrm{~cm}$ diameter. The piston can travel $5.6 \mathrm{~cm}$ over $24 \mathrm{sec}$ and has $1.2 \mathrm{~kg}$ lift capacity. The effect on buoyancy is $\Delta m=A \rho \Delta h$. The buoyancy system coordinates with the balance control mechanism (see Figure 3), which alters the center of mass of the robot by moving the battery pack up and down in the robot. The buoyancy system has an integrated docking mechanism (see Figure 2 and [1]) which enables the automatic pickup of payloads that are compatible with the docking mechanism (for example, our underwater sensor network nodes can be picked up by this robot). The buoyancy control module is contained in a water-tight cylindrical tube that can be attached to the robot with an underwater connector. It includes a piston moved by a set of three ball screws with ball nuts. A custom-designed gear box (Figure 2 (Bottom)) ensures that the ball screws are turned simultaneously and provides a gear ratio that can compensate for forces arising at up to $40 \mathrm{~m}$ depth 1 . The output power

\footnotetext{
${ }^{1}$ The force on the piston is about $10500 \mathrm{~N}$ at $40 \mathrm{~m}$ depth.
} 
$P_{\text {out }}$ of the motor is $P_{\text {out }}=\frac{\left(V-k_{r} \frac{T_{m}}{k_{m}}\right) k_{s} T_{m} 2 \pi}{60}$, where $V$ is voltage, $T_{m}$ is the motor torque, $k_{r}$ is the terminal resistance in $\Omega, k_{m}$ the torque constant in $\frac{m N m}{A}$ and $k_{s}$ as the speed constant in $\frac{R P M}{V}$. We found that the best performance was for gears of 64 teeth for the ball screws and 32 for the motor gear.

The balance control module is implemented as a moving battery inside the robot. We have developed a battery pack consisting of 72 Lithium-Ion cells that give the robot up to 8 hours of autonomous operation. This pack acts as a movable weight of 5.7 $\mathrm{kg}$. Shifting this weight inside the body of the robot (full time takes $30 \mathrm{sec}$ ) changes the center of mass. The battery system moves by sliding on four rails. Its position is controlled with a 20 turns per inch lead screw connected to a motor over a worm gear with a total 11:1 reduction. Figure 3 shows the different states of the robot with and without attached weight. The balance control system is not back-drivable and can compensate for up a weight of $1 \mathrm{~kg}$ attached to the tail of the robot. The balance system is especially important for maintaining the robot's angle when traveling in horizontal configuration.

\section{Control}

The robot posture and depth are controlled by $1 \mathrm{Khz}$ PD loops operating the 5 thrusters, using feedback from a custom-built 3D IMU. The buoyancy and balance control motors are operated with commands which are low passed filtered versions of the corresponding thrusters commands. This enables seemingly achieving neutral buoyancy, static balance in the desired posture, and efficient depth change. This way the robot can efficiently hover and transit with or without payload, with no additional thrust.

Suppose a payload of mass $m$ (in water) is attached to the robot at depth $d$ and carried for time $t$. If the robot uses thrusters only, the energy consumption is: $E=K_{p} \times m^{2} \times t$,

\section{- Thrusters}

$$
E=K_{p} \times m^{2} \times t
$$

- Buoyancy

$$
E=K_{b} \times m \times d
$$

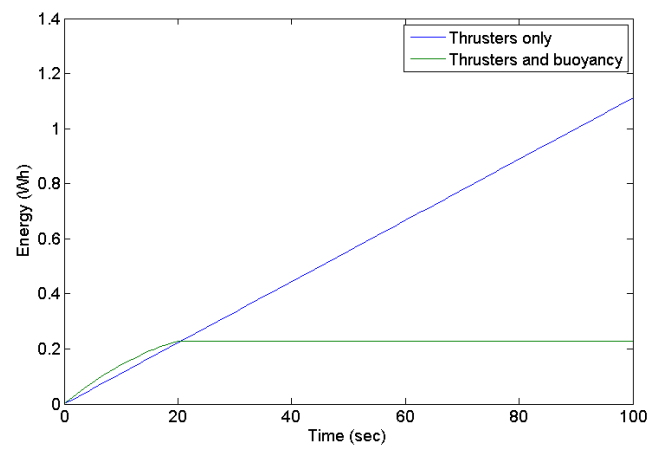

Fig. 4. Energy Analysis for a system that uses (a) thrusters (top curve) only and (b) thrusters and buoyancy engine (bottom curve). The x-axis shows the system's energy consumption in Wh. The y-axis shows time in sec. The simulations were done for a payload of $1 \mathrm{~kg}$ at $5 \mathrm{~m}$ depth. The constants used were $K p=40 \mathrm{~W} /\left(\mathrm{kg}^{2}\right)$ and $K b=160 \mathrm{~J} /(K g * m)$. 


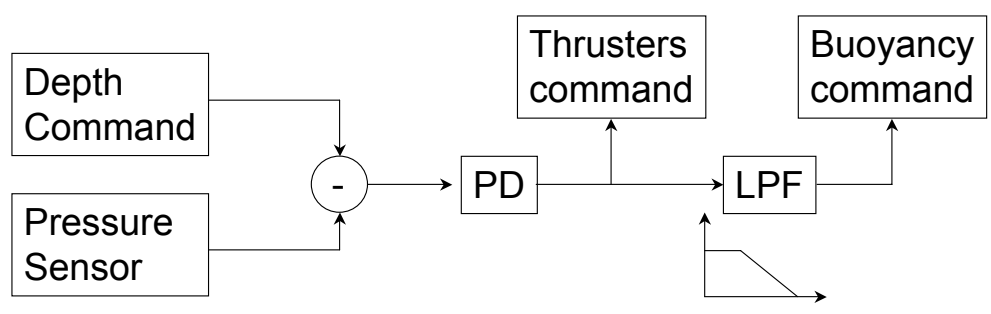

Fig. 5. The depth and buoyancy engine control loop

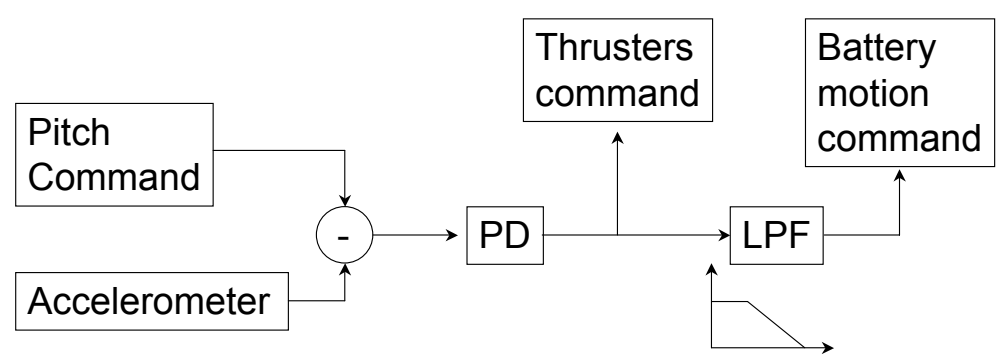

Fig. 6. The balance engine control loop

where $K_{p}$ is the a constant related to the thrusters efficiency (which is about $20 \mathrm{~W} / \mathrm{kg}^{2}$ in our system). The longer the payload is carried the more energy it is used. If instead the buoyancy engine is used the energy consumption is $E=K_{b} \times m \times d$, where $K_{b}$ is a constant related to our buoyancy engine efficiency (which is about $35 \mathrm{~J} /(\mathrm{kg} * \mathrm{~m}$ ) in out system). Figure 4 shows the trade-offs between using thrusters only and using thrusters and a buoyancy engine. The energy does not depend on the time the payload will be carried, but does depend on the depth at which is picked up. For example, for a $1 \mathrm{~kg}$ payload collected at $10 \mathrm{~m}$ depth the thrusters will use $20 \mathrm{~J} / \mathrm{sec}$ while the buoyancy engine will use $350 \mathrm{~J}$ so the break even point is $17.5 \mathrm{secs}$. For the first 17.5 seconds the thrusters are more efficient beyond that the buoyancy engine. But if the object is to be carried for $5 \mathrm{~min}$, the thrusters will use 20 times more energy than the buoyancy engine.

Figure 5 shows the control loop for the depth and buoyancy engine. The thrusters are controlled by a PD feedback loop that corrects for the desired depth. The buoyancy engine receives a low pass filtered version of the thruster command. The buoyancy engine moves the piston in the direction that brings the robot to neutral buoyancy (close to $0 \%$ ) thruster output.

Figure 6 shows the control loop for the battery engine. The thrusters are controlled by a PD feedback loop that corrects for the desired pitch. The battery motor receives a low pass filtered version of the thruster command. The battery is moved in the direction that brings the robot to neutral balance. 


\section{Experimental Results}

Several experiments were done to evaluate the buoyancy and balance control. The buoyancy experiment examines the aggregate thruster output as the robot's weight is changed by adding or removing a weight of $950 \mathrm{~g}$. Figure 7 shows the results. At time $t=0$ the robot hovers at constant depth in vertical configuration. The thrusters oscillate around $0 \%$ output. The robot's depth is maintained at $0.5 \mathrm{~m}(+/-2 \mathrm{~cm})$ below the water surface. The depth controller output oscillations are due to the latency of the sensorless motor controllers while changing direction (we note that the larger oscillations are due to human touch in preparation for adding a weight). At time $t=100 \mathrm{~s}$ a weight of $950 \mathrm{~g}$ is added to the robot. The depth controller oscillates till time $t=110 \mathrm{~s}$ in response to this event. Between times $t=110 \mathrm{~s}$ and $t=170 \mathrm{~s}$ the depth controller is stable at $40 \%$ output to compensate for the additional weight. We note that the oscillations are smaller in this phase since the controller does not need to change the direction of rotation as in the neutrally buoyant case. At time $t=170$ s the buoyancy engine is activated. Between $t=170 \mathrm{~s}$ and $t=185 \mathrm{~s}$ the buoyancy engine effects work to compensate for the additional weight of the robot. At time $t=185 \mathrm{~s}$ the robot is again neutrally buoyant and the depth controller operates around $0 \%$. At time $t=230$ s the robot releases the attached weight and the depth controller starts compensating for the positive buoyancy of the robot. At time $t=250$ s the buoyancy engine is activated again to bring the robot back to neutral buoyancy which happens at time $t=265 \mathrm{~s}$. We have repeated the buoyancy control experiment 10 times with similar results. The average time required to compensate for the robot's weight change is $15 \mathrm{~s}$.

The next group of experiments illustrates the effect of moving the battery inside robot on the pitch controller. Figure 8 shows the data. The robot starts in horizontal configuration with a $950 \mathrm{~g}$ weight attached to its bottom. The battery is at its bottom position in the robot's cylindrical enclosure. The thrusters work at $-40 \%$ to compensate for the weight. At time $t=10$ s the battery begins moving toward its top-most position.

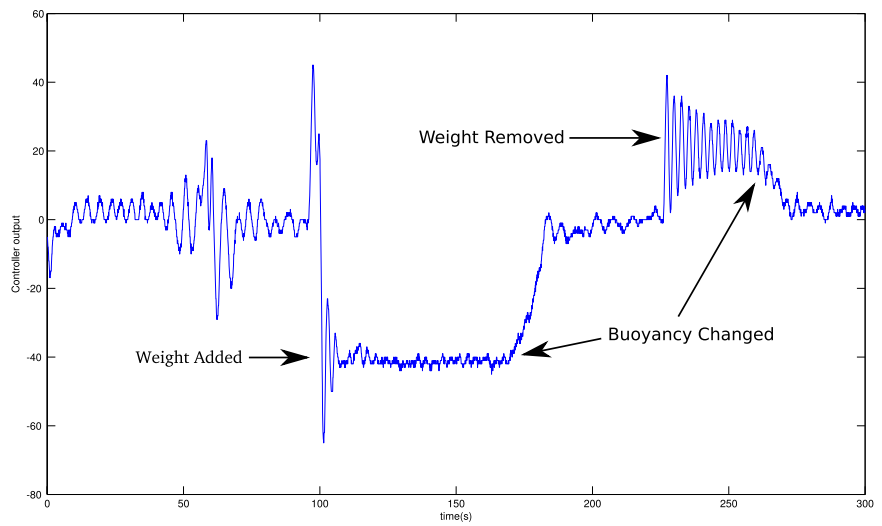

Fig. 7. Buoyancy experiment: thruster controller output (in percentage) over time as we vary the robot's weight 


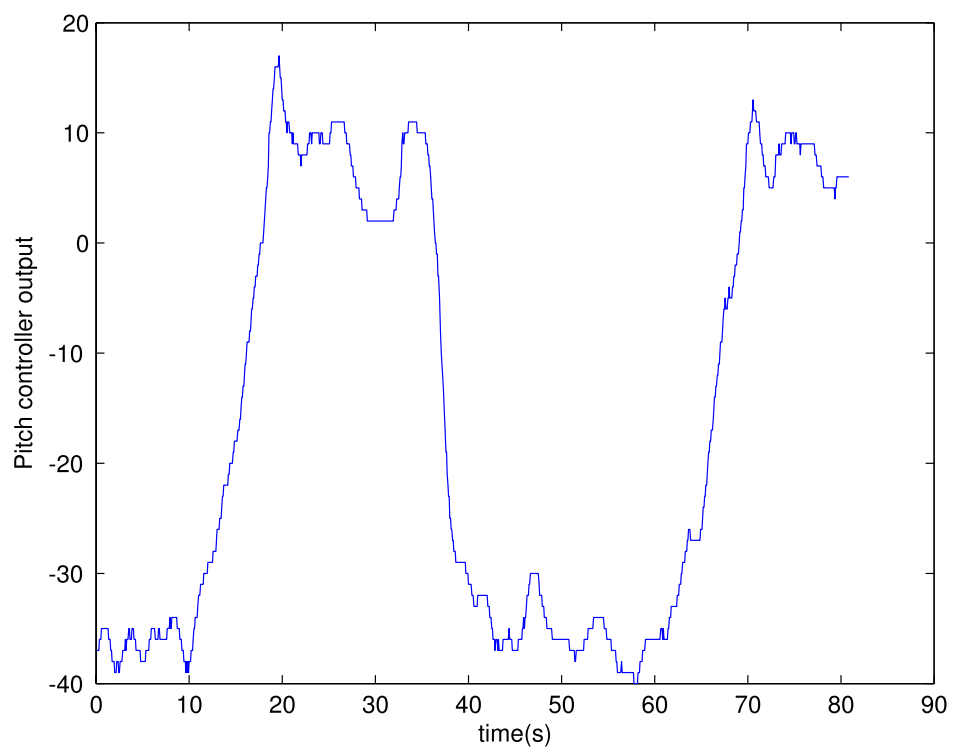

Fig. 8. Battery experiment: thruster controller output (in percentage) over time as we change the robot's balance by moving the battery's position

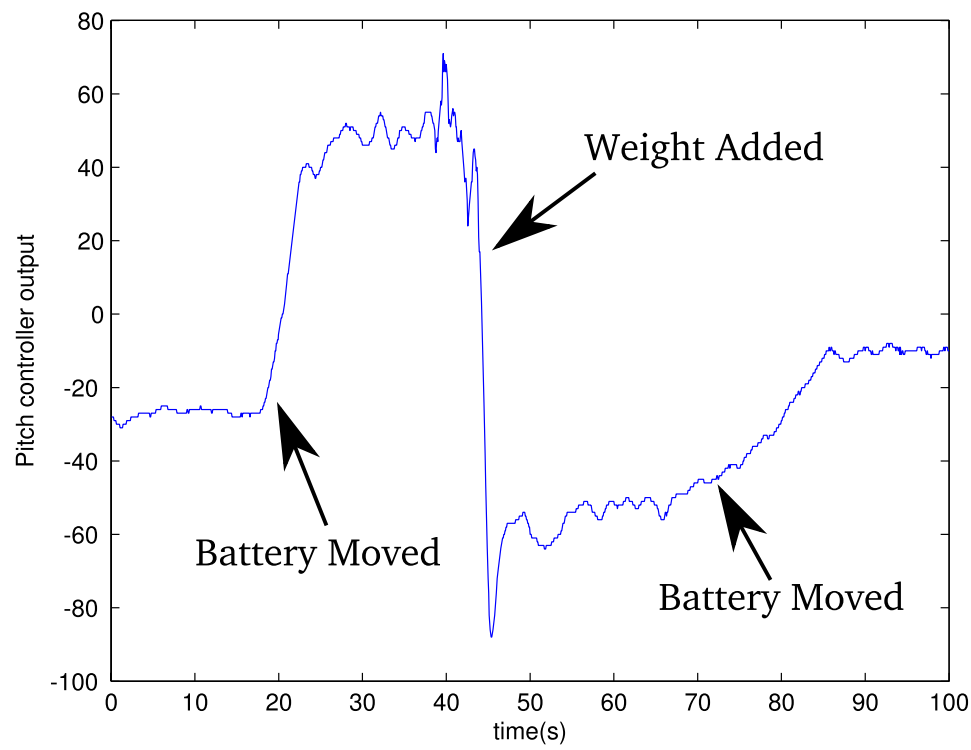

Fig. 9. Balance experiment: thruster controller output (in percentage) over time as we change the robot's balance by adding weight 
It takes 25 s to complete the move. We observe the controller output at $10 \%$ (in the other direction). This means that the robot's battery can compensate for more than the $950 \mathrm{~g}$ attached to it. The movement is repeated back and forth.

A third suite of experiments concerns balance control. Figure 9 shows the data. In these experiments the robot starts by hovering in horizontal configuration with the battery placed at the bottom of the robot's cylindrical body. The thrusters keep the robot hovering in horizontal stance. At time $t=20 \mathrm{~s}$ the battery is moved to its mid-point in the body of the robot and subsequently we observe the effect on the pitch controller's output. At time $t=45 \mathrm{~s}$ a weight of $950 \mathrm{~g}$ is added manually at the rear end of the robot and again we observe the effect on the pitch controller's output. At time $t=67 \mathrm{~s}$ the robot starts moving the battery autonomously to adjust the robot's balance. At time $t=85$ s we observe that the robot has successfully achieved neutral balance and subsequently the pitch controller oscillates around 0 . The balance control experiment was repeated 10 times with similar performance. The average time to achieve neutral balance was 20 s.

\section{Conclusions}

In this paper we described a robot that can adjust its static buoyancy and balance to save energy when its weight changes by picking up or dropping a payload during a mission. We described the hardware used to achieve this kind of adaptive control and the supporting algorithms. We showed experimentally that this system can reliably save energy with buoyancy and balance control. We believe that simultaneous buoyancy and balance control will enable underwater robots to carry out pick and place operations efficiently and plan to demonstrate this point in a new suite of experiments to deploy and reposition an underwater sensor network. These experiments will be carried out in the ocean at the Gump Marine Biology Research Center in August 2008.

\section{Acknowledgments}

We thank Daniel Gurdan and Jan Stumpf for their invaluable contributions to Amour's electronics. We thank Randy Davis for the use of his pool and to Tim Beaulieu for supporting our pool work. This work was supported in part by DSTA and the NSF. We are grateful for this support.

\section{References}

1. Dunbabin, M., Vasilescu, I., Corke, P., Rus, D.: Cooperative navigation experiments with two autonomous underwater robots (2006)

2. Gurdan, D.: Design and motion control of an autonomous underwater vehicle. Diploma thesis, TUM / MIT, Munich (January 2007)

3. Laine, J.L., Nichols, S.A., Novick, D.K., Malley, P.D.O., Copeland, D., Nechyba, M.C.: Subjugator: a highly maneuverable, intelligent underwater vehicle. Technical report, Machine Intelligence Laboratory, University of Florida, Gainesville, FL (1999) 
4. McFarland, D., Gilhespy, I., Honary, E.: Divebot: A diving robot with a whale-like buoyancy mechanism. Robotica 2003, 21(04) (August 2003)

5. Novick, D.K., Pitzer, R., Wilkers, B., Crane, C.D., de la Iglesia, E., Doty, K.L.: The development of a highly maneuverable underwater vehicle. In: Robotics 1998: The 3rd International Conference and Exposition/Demonstration on Robotics for Challenging Environments, Albuquerque, NM, April 1998, pp. 168-173 (1998)

6. Sherman, J., Davis, R.E., Owens, W.B., Valdes, J.: The autonomous underwater glider 'spray.' . In: IEEE Oceanic Engineering, vol. 26, pp. 437-446 (2001)

7. Stumpf, J.: Design and motion planning of an autonomous underwater vehicle. Diploma thesis, TUM / MIT, Munich (January 2007)

8. Vasilescu, I., Varshavskaya, P., Kotay, K., Rus, D.: Autonomous modular optical underwater robot (amour): design, prototype and feasibility study. In: Proceedings of the 2005 International Conference on Robotics and Automation, Barcelona (April 2005) 